\title{
Impact of intelligent phacoemulsification software on torsional phacoemulsification surgery
}

This article was published in the following Dove Press journal:

Clinical Ophthalmology

II September 2012

Number of times this article has been viewed

\section{Silay Canturk Ugurbas \\ Sinan Caliskan \\ Atilla Alpay \\ Suat Hayri Ugurbas}

Department of Ophthalmology, Bulent Ecevit University School of Medicine, Zonguldak, Turkey

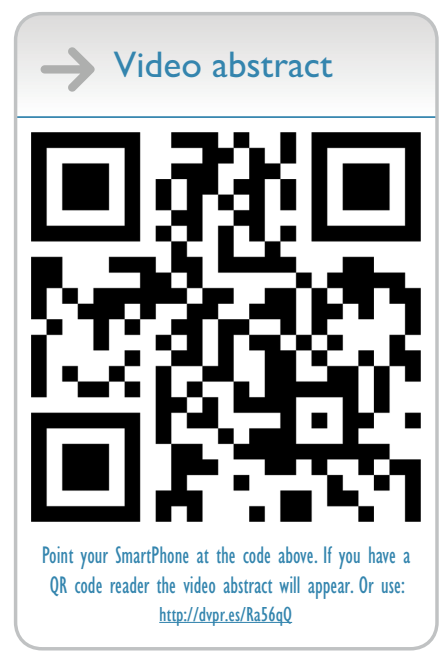

Correspondence: Silay Canturk Ugurbas Department of Ophthalmology, Bulent Ecevit University School of Medicine, Esenkoy, Zonguldak, 67600 Turkey

Tel +9037 226I 2265

Fax +9037226I 0155

Email silaycanturk@gmail.com
Background: The purpose of this study was to compare phacoemulsification energy parameters during torsional phacoemulsification with or without the use of intelligent phacoemulsification (IP) software.

Methods: One hundred and twenty-eight eyes with nuclear grades ranging from 1 to 5 were enrolled in this randomized prospective study. Operated eyes were divided into two groups, ie, those operated on using IP software (Group $1, n=67$ ) and those operated on without IP software (Group 2, $\mathrm{n}=61$ ). The two groups were compared in terms of ultrasound energy level, ultrasound energy time, aspiration time, and amount of fluid used during surgery.

Results: Operated eyes were further grouped according to soft (grade 1 and 2, $n=37$ ), medium (grade 3, $\mathrm{n}=46$ ), and hard (grade 4 and $5, \mathrm{n}=31$ ) nuclear densities. Both the study and the control groups were similar in distribution of nuclear density $(P>0.05)$. Cumulative dissipated energy was measured as $14.06 \pm 9.92$ in Group 1 and $14.22 \pm 10.99$ in Group $2(P=0.92)$. Total ultrasound time was 49 seconds in Group 1 and 52 seconds in Group $2(P=0.58)$. Although the torsional amplitude used was similar $(P=0.86)$ when IP was either on $(71 \%)$ or off $(68.4 \%)$, aspiration time was found to be 4 minutes 17 seconds in Group $1(P=0.86)$ and 5 minutes and 17 seconds in Group $2(P=0.007)$. Total fluid used was measured as $91 \mathrm{cc}(P=0.86)$ in Group 1 and $109 \mathrm{cc}(P=0.02)$ in Group 2.

Conclusion: The new IP software did not cause a difference in ultrasound energy levels. However, the new software was found to be advantageous in regards to fluid use and aspiration time.

Keywords: cataract surgery, phacoemulsification, Ozil, cumulative dissipated energy, ultrasound energy

\section{Introduction}

As the instruments used in modern phacoemulsification surgery have improved, cataract surgeons have become able to monitor the energy used during surgery. A cumulative dissipated energy (CDE) measurement is included in the Alcon Infiniti Vision System, which indicates the amount of energy released in the anterior segment during surgery. A low CDE indicates that less energy is transmitted to the cornea and that a faster recovery is expected. During longitudinal phacoemulsification, the phaco handpiece chops the nucleus into pieces by moving forward and backward, similarly to a jackhammer, but the energy efficiency is low because the forward and backward motion pushes the nuclear fragments away at the same time. With improvement of the hardware in the Infiniti phacoemulsification instrument (Alcon), chopping of the nucleus through rotatory and side-to-side movement of the phaco handpiece 
(torsional phacoemulsification) became possible. Torsional phacoemulsification works with the unique Ozil handpiece, and curved phaco tips are used instead of straight ones.

The objective of this approach is to reduce the power spent pushing fragments and increase the efficiency of the energy use through a side-to-side chopping motion. Studies comparing longitudinal phacoemulsification and torsional phacoemulsification have reported that less energy is used and that the phaco time is shorter in torsional phacoemulsification. ${ }^{1-3}$ A disadvantage of torsional phacoemulsification is the blockage that occurs when hard nuclear fragments are aspirated through the curved, narrow portion of the tip, especially when mini-flared or 30 degree Kelman tips are used. Intelligent phacoemulsification is a software program developed for torsional phacoemulsification to overcome this problem. With this software, longitudinal power with short pulses $(10 \mathrm{msec})$ is initiated when the vacuum reaches a predetermined level, and it continues until the vacuum level drops under the threshold level. In this manner, small bursts of longitudinal power are applied to emulsify the nuclear fragment further, enabling it to be pushed completely into the phaco tip. The surgery is expected to be performed largely in the torsional mode, and the longitudinal mode is applied only at times when the vacuum reaches the predetermined threshold. The purpose of this study was to research the effects of the Ozil intelligent phacoemulsification (IP) software on CDE, phaco time, volume of fluid used during surgery, and duration of the surgery.

\section{Materials and methods}

A total of 128 eyes from 119 consecutive patients operated on between November 2011 and February 2012 were enrolled after obtaining presurgical informed consent and approval from Bulent Ecevit University Faculty of Medicine Ethics Committee. The relevant surgical indication was finding a cataract that impairs vision.

Best corrected visual acuity and intraocular pressure measurements and anterior segment and dilated fundus examination using a slit lamp were performed for all of the patients. The power of the intraocular lens was calculated via the SRK/T formula. Topical anesthesia and the Alcon Infiniti Vision System with the Ozil phaco handpiece were used for cataract surgery. IP software settings were adjusted such that longitudinal phaco was applied when the vacuum reached $95 \%$ of the maximum level, with $10 \mathrm{msec}$ pulsation, and in a one-to-one ratio with torsional phaco power. The grades of cataract hardness were determined according to the level of difficulty of nucleus chopping during the surgery.
If the nucleus could be phacoaspirated easily and chopping was difficult to achieve, the cataract was classified as soft (grades 1 and 2); if the nucleus could immediately be divided into two pieces by chopping, it was classified as medium density (grade 3); and if dividing into two was not achieved immediately due to presence of a posterior plate, it was classified as hard (grades 4 and 5).

Cases for IP (using IP software) and non-IP (without the use of IP software) groups were determined randomly on the day of surgery using a blocked randomized scheme with computer generated numbers. The cataract surgery was performed by three senior cataract surgeons (SCU, AM, and SHU). All of the surgeons performed the surgery using the same ultrasound, vacuum and flow parameters and using the phaco chop or quick chop techniques. Topical anesthesia and the Alcon Infiniti Vision System with the Ozil phaco hand-piece were used, as was a $0.9 \mathrm{~mm}$ Kelman phaco tip with a 30 degree angle. Cumulative dissipated energy (CDE), ultrasound time, average torsional power, volume of fluid used, and aspiration time were read from the screen on the front panel of the device and recorded after the surgery was complete. CDE was calculated by the device through a formula that includes the phaco power and phaco time in the employed mode (IP or non-IP). In the non-IP (pure torsional) mode, $\mathrm{CDE}$ was calculated as mean torsional amplitude $\times$ torsional time $\times 0.4$. In the IP (mixed longitudinal and torsional) mode, $\mathrm{CDE}=($ mean phaco power $\times$ phaco time $)+($ mean torsional amplitude $\times$ torsional time $\times 0.4)$. The frequency of the phaco tip in torsional phaco was $80 \%$ of longitudinal phaco $(32 \mathrm{Kz}$ in torsional versus $40 \mathrm{Kz}$ in longitudinal) and the travel distance of the phaco tip in torsional phaco was $1 / 2$ of that in longitudinal phaco. This helped justify the coefficient of 0.4 .

Surgery was performed through a $2.75 \mathrm{~mm}$ clear corneal incision. Capsulorhexis was performed under 3\% sodium hyaluronate chondroitin sulfate viscoelastic support. Following hydrodissection and hydrodelineation, the nucleus was phacoemulsified by the quick chop or phaco chop techniques and aspirated. The cortical remnants were cleaned using bimanual irrigation and aspiration. Under viscoelastic support, a three-piece hydrophilic acrylic monofocal intraocular lens was implanted. After the viscoelastic material was aspirated, the incision was checked for leaks and surgery was terminated after assuring proper sealing of the wound. Traumatic cataract, uveitic cataract, cases in which a complication occurred during surgery, and cases with pseudoexfoliation or with small pupils were not included in the study. 
Continuous variables were expressed as the average, standard deviation, median, minimum and maximum values, and categorical variables were expressed as the frequency and percentage. Parametric testing assumptions for continuous variables in the study were analyzed using the Shapiro-Wilk test. Those variables not verifying parametric testing assumptions were compared using the Mann-Whitney U test between two groups and the Kruskal-Wallis test among three groups. In group comparisons of categorical variables, the Pearson Chi-square test was used. Grouping according to cataract grade were determined by the analysis of variance test. In the study, the comparisons with a $P$ value $<0.05$ were accepted as significant. The statistical analyses were performed using the SPSS 13.0 software package (SPSS Inc, Chicago, IL).

\section{Results}

Of the 119 patients 17 had diabetes mellitus, 42 had hypertension, 18 had coronary artery disease, and 8 had a history of stroke. The mean age of the patients was $67 \pm 9$ (range 42-88) years. The distributions of cataract grades were not found to be different between the groups using and not using IP ( $P=1.0$, Mann-Whitney U test). The distribution of cataract grades in both groups is shown in Table 1. The average CDE values showed a significant increase with respect to cataract grades ( $P=0.00$, analysis of variance). However, no difference in $\mathrm{CDE}$ was observed between the groups using and not using IP (Table 2).

The average ultrasound time spent was found to be 25 seconds in grade 1 and 2 cataracts, 52 seconds in grade 3 cataracts and one minute 9 seconds in grade 4 and 5 cataracts. Whereas this parameter exhibited a significant difference with respect to cataract grades $(P=0.00)$, no difference was observed between the groups using and not using IP $(P=0.58)$.

The average torsional phaco power was found to be $71.4 \%$ in the IP group and $68.4 \%$ in the non-IP group $(P=0.53)$. Whereas the average torsional phaco power was

Table I Distribution of cataract grades in groups operated with and without Ozil intelligent phacoemulsification software

\begin{tabular}{llll}
\hline $\begin{array}{l}\text { Cataract } \\
\text { grades }\end{array}$ & $\begin{array}{l}\text { Surgery with } \\
\text { IP software } \\
\text { (n) }\end{array}$ & $\begin{array}{l}\text { Surgery without } \\
\text { IP software } \\
\text { (n) }\end{array}$ & $\begin{array}{l}\text { Total } \\
\text { (n) }\end{array}$ \\
\hline I-2 (soft) & 14 & 18 & 32 \\
3 (medium) & 29 & 26 & 55 \\
$4-5$ (hard) & 24 & 17 & $4 \mid$ \\
Total (n) & 67 & $6 \mid$ & 128 \\
\hline
\end{tabular}

Abbreviation: IP, intelligent phacoemulsification.
Table 2 Cumulative dissipated energy levels in different cataract densities operated with or without Ozil intelligent phacoemulsification

\begin{tabular}{lllll}
\hline \multicolumn{5}{l}{ Mean CDE \pm SD } \\
\cline { 2 - 5 } & $\begin{array}{l}\text { Total } \\
(\mathbf{n}=\mathbf{I 2 8})\end{array}$ & $\begin{array}{l}\text { Grade I-2 } \\
(\mathbf{n}=\mathbf{3 2})\end{array}$ & $\begin{array}{l}\text { Grade 3 } \\
(\mathbf{n}=\mathbf{5 5})\end{array}$ & $\begin{array}{l}\text { Grade 4-5 } \\
(\mathbf{n}=\mathbf{4 1})\end{array}$ \\
\hline With IP & $14.0 \pm 9.9$ & $5.3 \pm 3.4$ & $12.5 \pm 6.9$ & $21.0 \pm 10.8$ \\
Without IP & $14.2 \pm 10.9$ & $4.9 \pm 3.9$ & $14.9 \pm 7.4$ & $23.1 \pm 12.9$ \\
$P$ value & 0.928 & 1.0 & 0.899 & 0.966 \\
\hline
\end{tabular}

Abbreviations: $\mathrm{CDE}$, cumulative dissipated energy; IP, intelligent phacoemulsification $\mathrm{SD}$, standard deviation.

found to be similar between grade 1 and $2(60.6 \% \pm 29.7 \%)$ cataracts and grade $3(70.7 \% \pm 27 \%)$ cataracts, a significant difference was observed when these two groups were compared with grade 4 and $5(76.3 \% \pm 25.8 \%)$ cataracts $(P=0.043)$.

The average surgery duration was found to be 11 minutes 35 seconds ( \pm 6 minutes 17 seconds) in the IP group and 12 minutes 28 seconds ( \pm 6 minutes 10 seconds) in the non-IP group $(P>0.05)$. Whereas the surgery duration was found to be similar between grade 1 and 2 (10 minutes 50 seconds) and grade 3 (10 minutes 52 seconds) cataracts, it was significantly longer in grade 4 and 5 (14 minutes 23 seconds) cataracts $(P=0.011)$. IP software did not result in a shorter case time for soft, medium, or hard cataract densities $(P=0.570$, $P=0.655$, and $P=0.458$, respectively).

Aspiration time was shorter in the IP group $(P=0.007$, Table 2). A subgroup analysis of aspiration time according to cataract density revealed that aspiration time was shorter in all nuclear densities if IP was utilized. However, this finding reached a statistically significant level for grade 4-5 cataracts only (Table 3 ).

The volume of fluid used in the group in which IP was applied was found to be $92 \pm 45 \mathrm{cc}$, whereas the amount in the group not using IP was $109 \pm 41 \mathrm{cc}(P=0.027)$. In both groups, the fluid volume used was found to increase with increasing cataract hardness; however, this trend was not found to be statistically significant $(P=0.127)$. The difference in the amount of fluid used increased with increasing cataract density in both the study and control groups, as shown in Figure 1. A subgroup analysis comparing the two surgeons using phaco chop (surgeon 1 and 2) with one surgeon (surgeon 3) using stop and chop revealed no significant difference in energy released during surgery, case time, aspiration time, or volume of fluid used. Comparison of IP eyes with non-IP eyes operated by the same surgeon also did not show a statistically significant difference (Table 4). 
Table 3 Mean aspiration time was found to be shorter in the study group operated using Ozil intelligent phacoemulsification software, and the difference in aspiration time was significant for hard cataract densities (grades 4-5)

\begin{tabular}{|c|c|c|c|c|}
\hline \multirow{2}{*}{$\begin{array}{l}\text { Mode of energy } \\
\text { delivery }\end{array}$} & \multicolumn{4}{|l|}{ Mean aspiration time \pm SD } \\
\hline & $\begin{array}{l}\text { Total } \\
(n=I \mid 4)\end{array}$ & $\begin{array}{l}\text { Grade I-2 } \\
(n=37)\end{array}$ & $\begin{array}{l}\text { Grade } 3 \\
(n=46)\end{array}$ & $\begin{array}{l}\text { Grade 4-5 } \\
(n=31)\end{array}$ \\
\hline With IP & $4 \mathrm{~min} 17 \mathrm{sec} \pm 2 \mathrm{~min} 13 \mathrm{sec}$ & $3 \min 58 \mathrm{sec} \pm 2 \min 4 \mathrm{sec}$ & $3 \min 48 \mathrm{sec} \pm 2 \min 10 \mathrm{sec}$ & $4 \min 55 \mathrm{sec} \pm 2 \min 16 \mathrm{sec}$ \\
\hline Without IP & $5 \mathrm{~min} 17 \mathrm{sec} \pm 2 \min 42 \mathrm{sec}$ & $4 \mathrm{~min} 38 \mathrm{sec} \pm 1 \mathrm{~min} 57 \mathrm{sec}$ & $4 \mathrm{~min} 44 \mathrm{sec} \pm I \min 50 \mathrm{sec}$ & $6 \mathrm{~min} 49 \mathrm{sec} \pm 1 \mathrm{~min} 48 \mathrm{sec}$ \\
\hline$P$ value & 0.007 & 0.379 & 0.062 & 0.007 \\
\hline
\end{tabular}

Abbreviations: IP, intelligent phacoemulsification; SD, standard deviation; min, minutes; sec; seconds.

\section{Discussion}

The results of this prospective study have shown that less volume of fluid is used and the aspiration time is shorter when the IP software is used, especially in hard cataract surgery. The use of curved Kelman phaco tips are reported to provide an advantage in torsional phaco surgery. ${ }^{4}$ However, in hard cataracts, curved tips may have a tendency to be blocked during the surgery. The observation of use of less fluid and shorter aspiration time in the group in which IP software was used, shows that the software may be advantageous in hard cataract surgery. These findings may indicate that with the use of this new software the occlusion of cataract particles in the tip is less interrupted and that the occlusion time is shorter. The operations were performed with less total energy use by IP software in medium and hard cataracts; however, the difference did not reach a statistically significant level.

There are several studies in the literature reporting that the IP software is advantageous. Cionni et al showed that the occlusion time, which they calculated using video overlay, was reduced in hard cataracts. ${ }^{5}$ In the same study, they observed that the amount of fluid used was also reduced. Shorter occlusion time during surgery protects against possible incision burns. Accordingly, IP software increases the

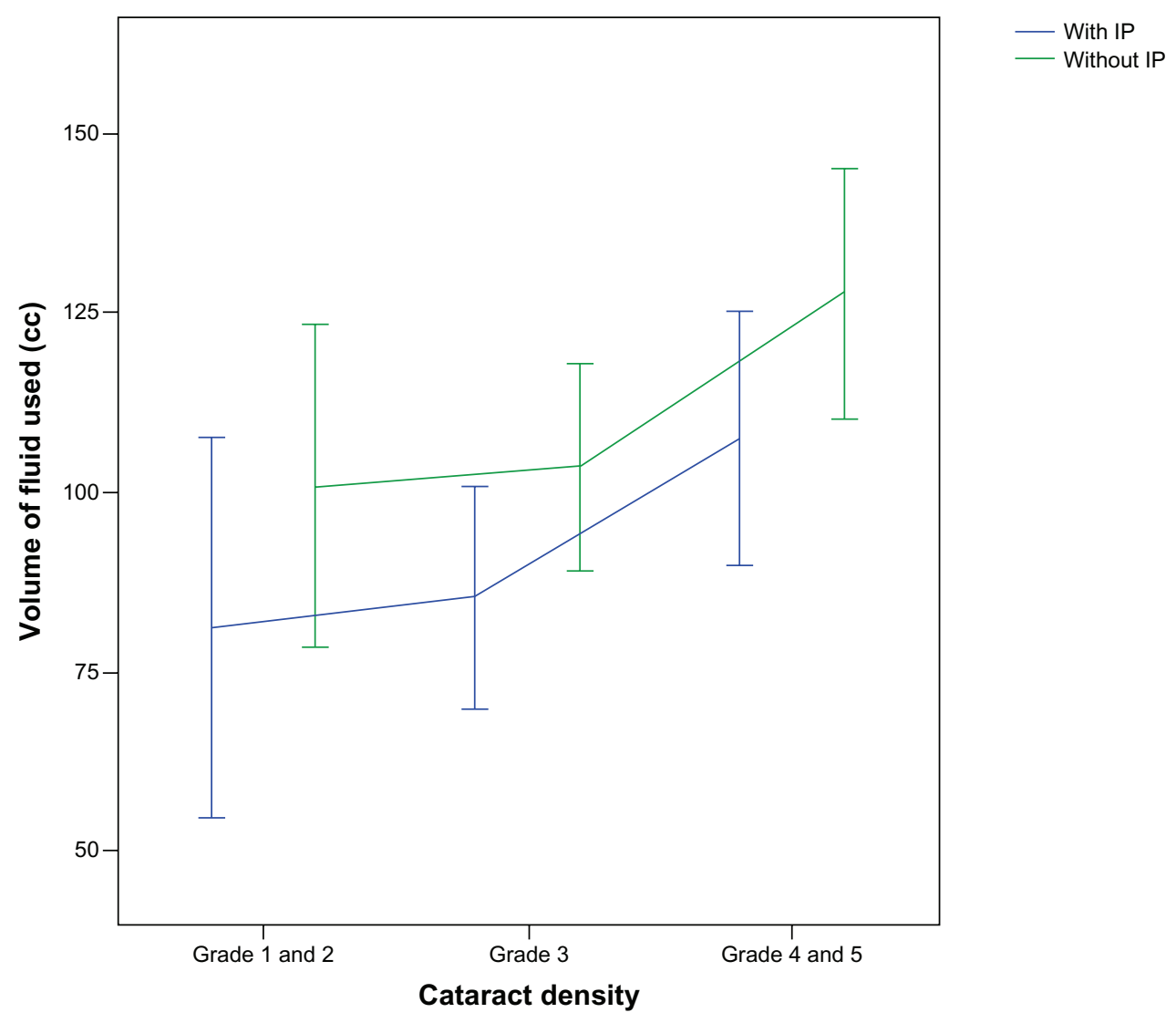

Figure I Changes in the amount of fluid used during surgery with or without IP (Intelligent Phacoemulsification) software with respect to cataract density. Note: Error bars: $\pm 2.0 \mathrm{SE}$. 


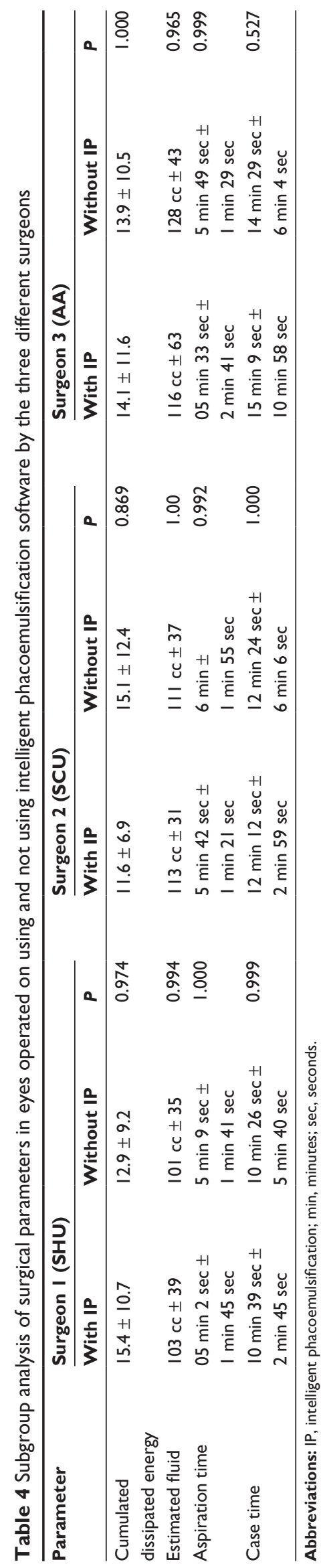

efficiency of surgery when torsional phaco is applied to hard cataracts.

In soft cataracts, the necessity of IP software is disputable. Zemba et al stated that the CDE is higher in soft cataracts when IP software is used. ${ }^{6}$ In our study, the CDE measured in grade 1-2 cataracts was 5.32 on average when IP was used and 4.87 when IP was not used. Even if the difference is not statistically significant, a reduced CDE is important, especially for patients with inadequate endothelial cell count. The greater CDE in soft cataracts when IP is used can be explained by the automatic transition to longitudinal phaco power, although the torsional power alone is sufficient for emulsification of nuclear fragments.

Ratnarajan et al used a stop and chop technique to divide the nucleus in half and compared the energy parameters of the half of the nucleus phacoemulsified with IP software turned on to the other half in which IP was chosen to be turned off. ${ }^{7}$ The energy released was measured to be significantly lower in the IP group in grade 4 and 5 cataracts. However, no statistically significant difference was found between grade 1, 2 , and 3 cataracts. In the same study, it was observed that IP software is not favorable in grade 2 cataracts.

It has been shown that pure longitudinal phaco releases twice the amount of energy that is released by pure torsional phaco. With IP software, the longitudinal ultrasound energy used during torsional phaco is limited. The use of this software is intended to chop the nucleus, shorten the occlusion time, and reduce the increase in heat to the minimum. Although a significant difference in energy parameters was not observed with the use of IP software in this study, we observed that IP software enables use of a smaller volume of fluid and a shorter aspiration time during phacoemulsification of hard cataracts. Further studies can be conducted to evaluate the correlations between these results and clinical variables including visual recovery and endothelium status.

\section{Disclosure}

The authors report no conflicts of interest in this work.

\section{References}

1. Reuschel A, Bogatsch H, Barth T, Wiedemann R. Comparison of endothelial changes and power settings between torsional and longitudinal phacoemulsification. J Cataract Refract Surg. 2010;36:1855-1861.

2. Rekas M, Montés-Micó R, Krix-Jachym K, Kluś A, Stankiewicz A, Ferrer-Blasco T. Comparison of torsional and longitudinal modes using phacoemulsification parameters. J Cataract Refract Surg. 2009;35: $1719-1724$.

3. Liu Y, Zeng M, Liu X, Luo L, Yuan Z, Xia Y et al. Torsional mode versus conventional ultrasound mode phacoemulsification: randomized comparative clinical study. J Cataract Refract Surg. 2007;33:287-292. 
4. Davison JA. Cumulative tip travel and implied followability of longitudinal and torsional phacoemulsification. $J$ Cataract Refract Surg. 2008;34:986-990.

5. Cionni RJ, Crandall AS, Felsted D. Length and frequency of intraoperative occlusive events with new torsional phacoemulsification software. J Cataract Refract Surg. 2011;37:1785-1790.
6. Zemba M, Cucu B, Furedi G, Enache V, Papadatu C, Ghigea B. Intelligent phaco - always necessary? Oftalmologia. 2011;55:68-73. Romanian.

7. Ratnarajan G, Packard R, Ward M. Combined occlusion-triggered longitudinal and torsional phacoemulsification during coaxial microincision cataract surgery: effect on 30-degree mini-flared tip behavior. J Cataract Refract Surg. 2011;37:825-829.

\section{Publish your work in this journal}

Clinical Ophthalmology is an international, peer-reviewed journal covering all subspecialties within ophthalmology. Key topics include: Optometry; Visual science; Pharmacology and drug therapy in eye diseases; Basic Sciences; Primary and Secondary eye care; Patient Safety and Quality of Care Improvements. This journal is indexed on

\section{Dovepress}

PubMed Central and CAS, and is the official journal of The Society of Clinical Ophthalmology (SCO). The manuscript management system is completely online and includes a very quick and fair peer-review system, which is all easy to use. Visit http://www.dovepress.com/ testimonials.php to read real quotes from published authors. 